\title{
SCIENTIFIC REPORTS

\section{OPEN MTHFR Ala222Val polymorphism and clinical characteristics confer susceptibility to suicide attempt in chronic patients with schizophrenia}

\begin{abstract}
Jia Hong Liu ${ }^{1}$, Cheng Zhu ${ }^{1}$, Ke Zheng ${ }^{1}$, Wei Tang ${ }^{1}$, Li Li Gao ${ }^{1}$, Tammy H. Trihn², Hanjing Emily Wu ${ }^{2}$, Da Chun Chen ${ }^{3}$, Mei Hong Xiu ${ }^{3 *}$ \& Xiang Yang Zhang ${ }^{4 *}$

Patients with schizophrenia (SCZ) exhibit higher suicide rates than the general population. However, the molecular mechanism responsible for the high rate of suicidal behavior in SCZ remains poorly understood. MTHFR Ala222 Val (C677T; rs 1801133) polymorphism has repeatedly demonstrated to play a pathological role in numerous mental disorders, but none of these studies focused on the susceptibility of suicidal behavior in SCZ. In the present cross-sectional study, we recruited 957 chronic inpatients with SCZ and 576 healthy controls to assess the psychopathological symptoms of SCZ and compare the frequency of the MTHFR Ala222Val genotype in both suicide attempters and non-attempters. Our results demonstrated no significant differences in MTHFR Ala222Val genotype and allele distributions between the SCZ patients and controls $(p>0.05)$, but showed a statistical significance in the distribution of Ala/Val genotype between suicide attempters and nonattempters $(p<0.05)$. Further logistic regression analysis showed that MTHFR Ala222Val genotype, psychopathological symptoms, number of cigarettes smoked per day and drinking status were related to suicide attempts in SCZ $(\mathrm{p}<0.05)$. Our study demonstrated that MTHFR Ala222Val polymorphism and some clinical characteristics might confer susceptibility to suicide in patients with SCZ.
\end{abstract}

Patients with schizophrenia (SCZ) are at high risk for attempting and completing suicide. Suicide is characterized by intentional termination of one's life, which is an important sequelae of chronic schizophrenia ${ }^{1,2}$. To date, suicide has been considered one of the most common causes of premature death in SCZ patients ${ }^{3}$. The average life expectancy of patients with SCZ is estimated to have decreased by approximately 14.6 years by suicide ${ }^{4}$. However, the pathophysiological mechanisms for the suicidality of SCZ patients are still unclear, particularly the biological pathological mechanism.

Evidence from twins, adoption, and family studies showed a significant increase of suicidal rates in suicidal probands ${ }^{5}$, which suggests that genetic risk factors may play an important role in suicidal behavior. The heritability of suicidal behavior is about $45 \%$, while environmental components account for $57 \%{ }^{6}$. Based on earlier genetic association studies focusing on several biological systems associated with suicidal attempts, a variety of potential candidate genes have been identified. For example, brain-derived neurotrophic factor (BDNF) and its receptor (NTRK2), which are highly expressed in the prefrontal cortex and hippocampus, have been shown as strong candidates for suicide; however, these results are controversial ${ }^{6}$. Also, another biological system involved in serotonergic transmission has been found to be candidate genes for suicidal behavior, including a tryptophan hydroxylase involved in synthesis, monoamine oxidase A involved in degradation, and a serotonin-1-A receptor involved in transmission ${ }^{7}$. Studies have demonstrated that nearly $90-98 \%$ of suicides are committed by people with some type of mental illness ${ }^{8}$. Thus, mental illness can be identified as the strongest risk factor for suicide. Among them, SCZ is one of the most common diseases associated with suicidal behavior ${ }^{9}$. Above evidences suggest that overlapping genetic risk factors are associated with suicidal behavior and psychopathology. However,

${ }^{1}$ Wenzhou Kangning Hospital, Wenzhou, China. ${ }^{2}$ Department of Psychiatry and Behavioral Sciences, The University of Texas Health Science Center at Houston, Houston, TX, USA. ${ }^{3}$ Beijing HuiLongGuan Hospital, Peking University, Beijing, China. ${ }^{4}$ CAS Key Laboratory of Mental Health, Institute of Psychology, Chinese Academy of Sciences, Beijing, China. *email: xiumeihong97@163.com; zhangxy@psych.ac.cn 


\begin{tabular}{|c|c|c|c|}
\hline Variable & $\operatorname{SCZ}(n=957)$ & HC $(n=576)$ & F or $\chi^{2}(p)$ \\
\hline Gender(male/female), n & $783 / 174$ & $263 / 313$ & $216.8(<0.001)$ \\
\hline Age (years) & $47.8 \pm 10.2$ & $45.8 \pm 12.8$ & $11.7(<0.01)$ \\
\hline Education (years) & $9.3 \pm 6.4$ & $8.7 \pm 3.2$ & $1.5(0.09)$ \\
\hline Smokers/Non-smokers, $\mathrm{n}$ & $627 / 318$ & $218 / 358$ & $117.7(<0.000)$ \\
\hline BMI $\left(\mathrm{kg} / \mathrm{m}^{2}\right)$ & $24.5 \pm 3.9$ & $25.1 \pm 3.9$ & $2.7(<0.01)$ \\
\hline Drinkers/Non-drinkers, $\mathrm{n}$ & $137 / 727$ & $121 / 455$ & $6.2(0.013)$ \\
\hline MTHFR genotype distribution & & & $2.0(0.36)$ \\
\hline $\mathrm{CCn}(\%)$ & $298(38.9)$ & $145(34.8)$ & \\
\hline CT n(\%) & $344(45.0)$ & $202(48.4)$ & \\
\hline TT n(\%) & $123(16.1)$ & $70(16.8)$ & \\
\hline \multicolumn{4}{|l|}{$M T H F R$ allele frequency } \\
\hline $\mathrm{C} / \mathrm{T}, \mathrm{n}$ & $590 / 940$ & $342 / 492$ & $0.08(0.83)$ \\
\hline
\end{tabular}

Table 1. Demographic, clinical and genetic data in patients with schizophrenia and healthy controls. Note: Data is given as mean \pm standard deviation; $\mathrm{BMI}=$ body mass index; $\mathrm{SCZ}=$ schizophrenia; $\mathrm{HC}=$ healthy control; MTHFR $=$ Methylenetetrahydrofolate reductase.

genetic markers associated with the etiology and pathophysiology of suicidal behavior in psychiatric patients, especially SCZ patients, are still unknown.

The MTHFR gene, located on chromosome 1 encodes a 5-methylenetetrahydrofolate reductase that catalyzes the reduction of 5,10-methylenetetrahydrofolate (methylene THF). Impaired MTHFR metabolism leads to hypomethylation of DNA, which increases the mutation ratio and leads to chromosome instability ${ }^{10}$ in addition to increased concentrations of potentially toxic homocysteine (Hcy) and abnormal concentrations of neurotransmitters $^{11}$. It was found that the substitution (C to T) of nucleotide 677 (rs1801133) in exon 4 of the MTHFR gene resulted in amino acid substitution (Ala222Val) and a reduction of MTHFR activity, which was most frequently studied in relation to mental disorders including SCZ ${ }^{12-14}$, autism spectrum disorders ${ }^{15-17}$, affective disorders ${ }^{18-20}$, anxiety disorders ${ }^{21,22}$ and alcohol dependence ${ }^{23}$. To the best of our knowledge, only one study has investigated whether the MTHFR Ala222Val polymorphism was associated with suicide in 692 victims of completed suicide and 3257 healthy controls. No association was found between the MTHFR Ala222Val polymorphism and sui$\operatorname{cide}^{24}$. In the study, only 16 of the 692 subjects were diagnosed with SCZ, and the diagnoses were based upon medical and forensic examinations of post-mortem rather than evaluations by psychiatrists. Recently, several striking meta-analyses have demonstrated that MTHFR Ala222Val polymorphism is significantly associated with $\mathrm{SCZ}^{13,25-28}$. In particular, three meta-analyses have shown a positive association between MTHFR Ala222Val polymorphism and SCZ in Asian population, but not in the European population ${ }^{13,28,29}$. Furthermore, the SCZ patients with the MTHFR Val/Val or Ala/Val genotype were found to have higher Hcy levels than those with the Ala/Ala genotypes, which might be associated with the increased risk of $S C Z^{30,31}$. A recent meta-analysis also indicated that increased plasma total Hcy levels may be associated with a higher risk of SCZ ${ }^{27}$. Interestingly, one study showed that MTHFR Val/Val genotype affected gray matter density and memory impairment in SCZ patients in a Chinese population ${ }^{32}$. Taken together, these studies have suggested that the MTHFR gene polymorphisms, especially the MTHFR Val/Val genotype, may be involved in the psychopathology of suicidality in SCZ patients.

Considering the high suicide rate of SCZ patients, the pathogenic role of the MTHFR gene, and the higher Hcy level in SCZ, it would be interesting to investigate the relationship between the functional polymorphism of MTHFR Ala222 Val and suicide attempt of SCZ patients in a genetically more homogeneous Han Chinese population. Based on previous literature, we hypothesized that the Val allele carriers of the MTHFR Ala222Val would be associated with increased risk for suicide attempt in SCZ and may interact with some relevant clinical risk factors. We then examined whether the interaction of genetic and environmental factors might affect the risk of attempted suicide in SCZ patients.

\section{Results}

The demographic and clinical characteristics are shown in Tables 1 and 2. There were significant differences in sex, age, smoking status, and BMI between patients and healthy controls (all $\mathrm{p}<0.01$ ), which were controlled in the following analyses as confounding factors. The Ala222Val allelic and genotypic frequencies of SCZ patients and healthy controls are shown in Table 1. Distributions of the MTHFR Ala222Val genotypes were consistent with the Hardy-Weinberg equilibrium in both SCZ patients and healthy controls (both $\mathrm{p}>0.05$ ). Further, there were no significant differences in the Ala222Val allelic and genotypic distributions between SCZ patients and healthy controls (both $\mathrm{p}>0.05$ ). After adjusting for sex, smoking, BMI, and age, we still did not find any differences in the Ala222Val allelic and genotypic frequencies between the two groups (both $\mathrm{p}>0.05$ ). In addition, we did not find any association between the MTHFR Ala222Val genotypes and the positive and negative syndrome scales ${ }^{33}$ (PANSS) total and its 3 subscale scores (all p $>0.05$ ).

The SCZ patients included $152(15.9 \%)$ suicide attempters and 805 non-attempters. The Ala222Val allelic and genotypic frequencies of suicide attempters and non-attempters of patients are shown in Tables 2 and 3. On the level of genotypes, there was a significant difference between attempters and non-attempters $\left(\chi^{2}=4.7, \mathrm{df}=2\right.$, $\mathrm{p}=0.039)$, and the Ala/Val genotype was less frequent in attempters than in non-attempters (36.1\% vs $46.6 \%)$ in the codominant models (Ala/Val vs. Val/Val). The MTHFR Ala/Val genotype was associated with a lower suicide 


\begin{tabular}{|c|c|c|c|}
\hline Variable & $\begin{array}{l}\text { Attempters } \\
(\mathrm{n}=125)\end{array}$ & $\begin{array}{l}\text { Non-attempters } \\
(n=642)\end{array}$ & F or $\chi^{2}(p)$ \\
\hline Gender (male/female), $n$ & $105 / 20$ & $522 / 120$ & $0.51(0.48)$ \\
\hline Age (year), $\mathrm{n}(\%)$ & & & $4.2(0.13)$ \\
\hline$\leqq 30$ & $11(8.8)$ & $36(5.6)$ & \\
\hline $30-60$ & $91(72.8)$ & $442(68.8)$ & \\
\hline$\geqq 60$ & $23(18.4)$ & $164(25.6)$ & \\
\hline Education (year) & $8.9 \pm 2.9$ & $9.3 \pm 6.9$ & $0.75(0.45)$ \\
\hline Drinkers/Nondrinkers, $\mathrm{n}$ & $35 / 90$ & $91 / 676$ & $23.1(0.000)$ \\
\hline Age of smoking onset (year) & $19.2(3.1)$ & $21.9(4.0)$ & $2.51(0.015)$ \\
\hline Smoking status, $\mathrm{n}(\%)$ & & & $0.52(0.47)$ \\
\hline Smokers & $81(64.8)$ & $394(61.4)$ & \\
\hline Non-smokers & $44(35.2)$ & $248(38.6)$ & \\
\hline Marital status, n(\%) & & & $3.1(0.38)$ \\
\hline Single & $84(67.2)$ & $381(59.3)$ & \\
\hline Married & $22(17.6)$ & $130(20.2)$ & \\
\hline Divorced & $18(14.4)$ & $122(19.0)$ & \\
\hline Widowed & $1(0.8)$ & $9(1.4)$ & \\
\hline BMI $\left(\mathrm{kg} / \mathrm{m}^{2}\right)$ & $24.4 \pm 3.9$ & $24.6 \pm 4.0$ & $0.46(0.65)$ \\
\hline Age of illness onset (year) & $23.7(6.5)$ & $23.5(5.5)$ & $0.31(0.76)$ \\
\hline Duration of illness (year) & $24.8 \pm 9.9$ & $21.1 \pm 9.9$ & $4.2(0.000)$ \\
\hline Antipsychotic dose (CPZ) & $472.3 \pm 536$ & $452.2 \pm 392$ & $0.28(0.60)$ \\
\hline \multicolumn{4}{|l|}{ PANSS factors (score) } \\
\hline Positive & $8.7 \pm 74.8$ & $7.7 \pm 4.9$ & $4.7(0.03)$ \\
\hline Negative & $19.2 \pm 7.6$ & $18.9 \pm 6.4$ & $0.15(0.70)$ \\
\hline Cognitive & $7.5 \pm 3.0$ & $8.1 \pm 3.5$ & $3.5(0.06)$ \\
\hline Depression & $9.2 \pm 5.2$ & $7.9 \pm 5.2$ & $7.4(0.000)$ \\
\hline Excitement & $4.9 \pm 2.2$ & $4.6 \pm 1.7$ & $4.2(0.04)$ \\
\hline $\begin{array}{l}\text { MTHFR allele distribution, } \\
\mathrm{n}(\%)\end{array}$ & & & $0.09(0.83)$ \\
\hline Ala & $153(62.3 \%)$ & $787(61.3 \%)$ & \\
\hline Val & $97(37.7 \%)$ & $497(38.7 \%)$ & \\
\hline
\end{tabular}

Table 2. Demographic, clinical and genetic data in patients with schizophrenia divided with regard to suicide attempts. Note: Data is given as mean \pm standard deviation; $\mathrm{BMI}=$ body mass index; PANSS $=$ Positive and negative syndrome scale; $\mathrm{CPZ}=$ Chlorpromazine equivalent dose; $\mathrm{MTHFR}=$ Methylenetetrahydrofolate reductase.

\begin{tabular}{|c|c|c|c|c|c|c|}
\hline Genotype & Attempers $n=125$ & Nonattempters $n=642$ & p-value ${ }^{\mathrm{a}}$ & Crude OR (95\% CI) & Adjusted OR (95\% CI) & p-value ${ }^{b}$ \\
\hline \multicolumn{7}{|c|}{ MTHFR Ala222Val $(\mathrm{HWE}=0.791)$} \\
\hline $\mathrm{Val} / \mathrm{Val}$ & 26 & 99 & & 1 & 1 & \\
\hline $\mathrm{Ala} / \mathrm{Val}$ & 45 & 299 & 0.039 & $0.61(0.35-1.01)$ & $0.36(0.23-0.59)$ & 0.0000 \\
\hline $\mathrm{Ala} / \mathrm{Ala}$ & 54 & 244 & 0.52 & $1.10(0.64-1.87)$ & $0.97(0.73-1.28)$ & 0.83 \\
\hline
\end{tabular}

Table 3. Multiple logistic regression analysis of the MTHFR Ala222Val polymorphism and suicide susceptibility. Notes: ${ }^{a} \mathrm{Chi}$-squared tests were used to determine differences in genotype distributions between attempters and nonattempters. ${ }^{b}$ Adjusted for age, gender, smoking, BMI, drinking and marital status.

risk compared to the Val/Val genotype $(\mathrm{OR}=0.36,95 \% \mathrm{CI}=0.23-0.59$, adjusted $\mathrm{p}=0.000$; Table 3$)$ in patients with SCZ. There was no significant difference in the distribution of alleles between the two groups $\left(\chi^{2}=0.09\right.$, $\mathrm{df}=1, \mathrm{p}=0.83$ ).

We further analyzed the characteristics related to suicide and found significant differences in drinking status $(\mathrm{p}<0.001)$, onset age of smoking $(\mathrm{p}<0.05)$, cigarettes smoked each day $(\mathrm{p}<0.05)$, duration of illness $(\mathrm{p}<0.01)$, positive factor of PANSS $(\mathrm{p}<0.05)$, depression factor $(\mathrm{p}<0.001)$, and excitement factor $(\mathrm{p}<0.05)$ between attempter and non-attempter groups, as shown in Table 4 . However, only the difference in drinking status and depression factor of PANSS remained significant after the Bonferroni correction test (corrected $\mathrm{p}<0.05)$. There was no significant difference between the suicide attempters and non-attempters in age, gender, BMI, age of onset, marital status, and antipsychotic types and doses (in chlorpromazine equivalents) (all p $>0.05$ ).

In the binary logistic regression model for associations with suicide attempt, education $(\mathrm{OR}=0.89,95 \% \mathrm{CI}$ $[0.82,0.98]$, Wald $\left.\chi^{2}=5.6, \mathrm{p}=0.018\right)$, PANSS depression factor $\left(\mathrm{OR}=1.08,95 \% \mathrm{CI}[1.03,1.13]\right.$, Wald $\chi^{2}=11.04$, 


\begin{tabular}{|c|c|c|c|c|c|c|}
\hline \multirow[b]{2}{*}{ Variable } & \multicolumn{3}{|l|}{ Attempter } & \multicolumn{3}{|l|}{ Nonattempter } \\
\hline & Ala/Ala & Ala/Val & Val/Val & Ala/Ala & Ala/Val & Val/Val \\
\hline Gender (male/female), $\mathrm{n}$ & $32 / 6$ & $59 / 5$ & $17 / 5$ & $212 / 48$ & $240 / 40$ & $82 / 19$ \\
\hline Age (year) & $45.6 \pm 9.7$ & $45.6 \pm 9.7$ & $47.1 \pm 10.1$ & $49.0 \pm 9.4$ & $48.1 \pm 9.3$ & $47.9 \pm 9.6$ \\
\hline Education (year) & $9.3 \pm 2.3$ & $7.9 \pm 2.5$ & $8.6 \pm 2.6^{*}$ & $9.3 \pm 2.5$ & $9.3 \pm 5.8$ & $8.5 \pm 2.5$ \\
\hline Drinkers/Nondrinkers, $\mathrm{n}$ & $3 / 18$ & $20 / 23$ & $11 / 39^{*}$ & $13 / 76$ & $31 / 243$ & $34 / 189$ \\
\hline Cigarette/day & $27.4 \pm 5.6$ & $23.9 \pm 4.5$ & $21.3 \pm 3.8^{*}$ & $22.5 \pm 4.8$ & $19.5 \pm 10.3$ & $18.8 \pm 2.0$ \\
\hline Smokers/Non-smokers, $\mathrm{n}$ & $17 / 7$ & $32 / 13$ & $36 / 18$ & $62 / 37$ & $180 / 118$ & $167 / 76$ \\
\hline $\operatorname{BMI}\left(\mathrm{kg} / \mathrm{m}^{2}\right)$ & $24.9 \pm 3.9$ & $25.4 \pm 4.3$ & $23.6 \pm 3.7$ & $24.7 \pm 4.1$ & $24.6 \pm 3.9$ & $24.6 \pm 3.9$ \\
\hline Age of onset (year) & $25.0 \pm 9.3$ & $23.1 \pm 4.8$ & $23.8 \pm 6.3$ & $23.4 \pm 6.0$ & $23.5 \pm 5.4$ & $23.2 \pm 4.8$ \\
\hline Duration of illness (year) & $20.0 \pm 8.9$ & $22.3 \pm 8.7$ & $23.4 \pm 9.6$ & $25.6 \pm 9.2$ & $24.6 \pm 9.8$ & $24.6 \pm 9.1$ \\
\hline Antipsychotic dose (CPZ) & $459.5 \pm 464.0$ & $402.9 \pm 175.4$ & $509.7 \pm 403.2$ & $446.9 \pm 311.9$ & $478.0 \pm 448.7$ & $421.3 \pm 270.1$ \\
\hline \multicolumn{7}{|l|}{ PANSS factors (score) } \\
\hline Positive & $8.7 \pm 4.8$ & $8.8 \pm 4.1$ & $8.9 \pm 5.1$ & $7.9 \pm 4.9$ & $7.4 \pm 4.9$ & $7.0 \pm 4.3$ \\
\hline Negative & $19.6 \pm 5.2$ & $18.8 \pm 6.5$ & $20.9 \pm 6.3$ & $3.6 \pm 1.2$ & $3.5 \pm 1.2$ & $3.6 \pm 1.2$ \\
\hline Cognitive & $7.7 \pm 3.0$ & $7.5 \pm 2.6$ & $8.4 \pm 3.1$ & $8.2 \pm 3.5$ & $7.9 \pm 3.6$ & $8.1 \pm 3.4$ \\
\hline Depression & $5.0 \pm 2.6$ & $4.3 \pm 1.9$ & $4.7 \pm 2.3$ & $3.6 \pm 1.2$ & $3.5 \pm 1.2$ & $3.6 \pm 1.2$ \\
\hline Excitement & $4.6 \pm 0.9$ & $5.1 \pm 2.7$ & $5.2 \pm 2.5$ & $4.7 \pm 2.0$ & $4.6 \pm 1.8$ & $4.6 \pm 1.5$ \\
\hline
\end{tabular}

Table 4. Demographic and clinical data in patients with schizophrenia divided with regard to suicide attempt and MTHFR Ala222Val genotypes. Note: Data is given as mean \pm standard deviation; BMI = body mass index; PANSS $=$ Positive and negative syndrome scale; $\mathrm{CPZ}=$ Chlorpromazine equivalent dose; MTHFR $=$ Methylenetetrahydrofolate reductase. ${ }^{*} \mathrm{p}<0.05$.

$\mathrm{p}=0.001)$, number of cigarettes smoked per day $\left(\mathrm{OR}=1.20,95 \% \mathrm{CI}[1.18,1.52]\right.$, Wald $\left.\chi^{2}=4.9, \mathrm{p}=0.043\right)$, and drinking status $\left(\mathrm{OR}=0.40,95 \% \mathrm{CI}[0.24,0.66]\right.$, Wald $\left.\chi^{2}=13.0, \mathrm{p}<0.001\right)$ remained significant and MTHFR Ala222Val polymorphism $\left(\mathrm{OR}=1.72,95 \% \mathrm{CI}[0.94,3.2]\right.$, Wald $\left.\chi^{2}=3.1, \mathrm{p}=0.07\right)$ approached significance.

The whole sample in the current study had a power of 0.99 to detect the association of MTHFR Ala222Val with SCZ as dominant, recessive, and log additive inheritance, with an OR of 2 ( $\alpha=0.05$, two-tailed test). Moreover, the suicide attempt sample had $0.88-0.99$ statistical power to detect this polymorphism to have relationship with suicide attempts as dominant, recessive, and log additive inheritance, with an OR of 2 ( $\alpha=0.05$, two-tailed test).

\section{Discussion}

There are three main findings in the present study. First, we did not find any significant difference in the MTHFR Ala222Val polymorphism between the patients with SCZ and healthy controls, which was in accordance with a couple of previous studies ${ }^{34-36}$, suggesting that the Ala222Val polymorphism of the MTHFR gene did not contribute to increased risk of SCZ in our Chinese samples. Second, the MTHFR Ala222Val polymorphism appears to be weakly correlated with suicide attempts in SCZ. Moreover, compared to patients without lifetime suicide attempts, the Ala/Val genotype was significantly less frequent and the Val/Val genotype was more frequent in lifetime suicide attempts of SCZ patients (35.8\% vs $46.6 \%, 20.3 \%$ vs $15.4 \%$ ). Third, in addition to MTHFR Ala222Val polymorphism, higher psychopathological symptoms and more severe depressive symptoms, more cigarettes smoked, and more drinking were significantly correlated to suicidal risk, suggesting that a multifactorial etiology involving both genetic heritability and environmental factors may contribute to suicidal behaviors among SCZ patients.

Numerous studies have investigated the possible role of functional polymorphism of MTHFR gene in the risk of pathogenesis of SCZ, but the results are inconsistent. We found that MTHFR Ala222Val polymorphism did not play a significant role in the pathogenesis of SCZ in our current study, which is consistent with several previous studies in Iranian, Korean, and Nordic populations ${ }^{37}$. However, the most recent meta-analysis reported a positive association between Ala222 Val and SCZ in eastern $\mathrm{Asia}^{27}$, which is completely different from our results. A possible reason for the inconsistencies in these genetic association studies may be due to ethnic background. For example, the genotype frequency distribution of Ala222Val is significantly different between the Chinese and European populations. The frequency of Val/Val genotype in healthy controls was approximately $16 \%$ in the Chinese population, but only about $9 \%$ in the European population. Therefore, interethnic differences in the genotype frequencies of the MTHFR Ala222Val polymorphism may lead to inconsistent results across the different populations. Other factors may also contribute to these controversial results, such as small or moderate genetic effects of Ala222Val, diet with increased folate consumption, relatively small sample sizes, heterogeneity in SCZ diagnosis, and gender stratification.

To our best knowledge, this is the first study to report the positive association between the MTHFR Ala222Val polymorphism and suicidal behavior in SCZ patients. Moreover, no significant association was found between the MTHFR Ala222Val genotypes and the PANSS total and its 3 subscale scores (all $\mathrm{p}>0.05$ ), suggesting that the MTHFR Ala222Val polymorphism appears not to be a general marker of a more severe disease but rather specific for suicide. Only one previous study investigated the association between suicide and Ala222Val polymorphism in postmortem and reported contradictory results ${ }^{24}$. A possible explanation for the inconsistency in results is that the subjects enrolled in the present study were suicide attempters and not suicide completers. 
In addition, different methods were used in the diagnosis of SCZ (long-term chronic hospitalization of SCZ patients vs no psychological autopsy). Thus, the differences in the participants may account for the discordant results in these two studies. Our current study suggested that MTHFR Ala/Val genotype may lay a critical role in suicidal behaviors. The $222 \mathrm{Val}$ allele was considered to be a mutant allele. Ala222 Val polymorphism was thought to be associated with alteration of MTHFR enzyme activity as the heterozygous genotype (Ala/Val) and mutant homozygous genotype (Val/Val) have only $67 \%$ and $25 \%$ of the MTHFR enzyme activity of their Ala/Ala wild type counterparts, respectively ${ }^{22}$. In the present study, the Val/Val genotype was over-present and the Ala/Val genotype was less present among suicide attempters in patients with SCZ. We speculate that the association of MTHFR Ala/Val genotype with suicide attempt may be in relation to the reduced MTHFR enzyme activity. A reduction in MTHFR enzyme activity results in a decline in its ability to irreversibly reduce 5,10-methylene THF to 5-methyltetrahydrofolate (5-methyl THF) and produce methyl groups involved in epigenetic regulation ${ }^{22}$. The declined enzyme activity may either raise the concentration of potentially toxic Hcy in those with inadequate dietary folate intake, or lead to hypomethylation of DNA ${ }^{38,39}$. The studies in MTHFR gene knockout mice have shown that plasma total Hcy was 10-fold and 1.6-fold higher, respectively, in MTHFR - /- and MTHFR +/- mice than in wild-type controls $s^{40,41}$. Numerous studies have also demonstrated that elevated total Hcy levels in plasma are one of the most important risk factors for psychiatric diseases such as depression and SCZ ${ }^{42-45}$, although few studies have focused on the direct relationship between suicide attempt and Hcy in SCZ. However, it is worth mentioning that this explanation for the association between heterozygous genotypes and suicide attempts may stem from the enzymatic effects of genotypes, which is only our speculation. If our interpretation in this part was of major importance, there should be an even stronger difference between the wildtype (Ala/Ala) and the homozygous mutant genotype ( Val/Val) than between Ala/Ala and Ala/Val genotypes, and the difference should also be obvious when alleles were compared. However, the fact is that the Ala/Ala and Val/Val genotypes were almost equally present and far from significantly different after clinical variables were controlled (Table 3). Moreover, there was no significant difference in allele frequencies between attempters and non-attempters. Taken together, this interpretation for the association between heterozygous genotypes and suicide attempts due to the enzymatic effects of genotypes is quite speculative and may provide only part of the reasons. However, there are some other important mechanisms underlying the association between heterozygous genotypes and suicide attempts; for example, proximal variants or genes that may be in linkage disequilibrium with the MTHFR Ala/ Val polymorphism may also be associated with suicide attempt in schizophrenia. Therefore, the mechanism linking the MTHFR Ala/Val gene polymorphism and attempted suicide is still unknown, which warrants further investigation.

It is well known that the severity of depressive symptoms was the most robust risk factor of suicidal behaviors in psychiatric disease $e^{46,47}$. Accordingly, a study of long-term hospitalized SCZ patients in China found that $72 \%$ of suicidal attempts were accompanied by depressive symptoms ${ }^{48}$. Further, the $222 \mathrm{Val} /$ Val genotype was found to be associated with depression, anxiety, and cognitive impairment, which all had close relationships with suicidal behavior in later life ${ }^{49}$. Since the interaction of Hcy and depressive symptoms play a pathogenic role in suicidal behaviors, we presumed that increased Hcy, as a consequence of the mutant genotype in embryonic neural cells, exerts neurotoxic effects, resulting in neonatal neurodevelopmental defects related to suicidal behavior $^{50,51}$. Indeed, studies found that high Hcy levels lead to abnormal levels of monoamine neurotransmitters, such as 5-serotonin and dopamine and cause aberrant BDNF level ${ }^{52-54}$, which may all participate in the occurrence of depressive symptoms related closely to suicide behavior in SCZ. On the other hand, abnormal epigenetic modifications caused by dysfunctional enzyme activity during pregnancy, such as DNA hypomethylation, are increasingly recognized to have lasting developmental implications with regard to suicide behavior ${ }^{55,56}$. Evidence supports that hypomethylation of DNA could increase the mutation ratio and cause chromosome instability with neurodevelopmental consequences due to downstream effects on dopaminergic and serotonergic systems important for developmentally stable emotional, cognitive, and behavioral phenotypes in childhood and may increase suicidal behaviors later in life $\mathrm{e}^{57}$. However, it is worthy of mentioning that although there was association between the 222Val/Val genotype and depression, anxiety, and cognitive impairment, which are conditions associated with suicidal behavior ${ }^{49}$, our results indicated association of attempted suicide with the Ala/Val genotype but not with Val/Val genotype, suggesting a heterozygous effect of the MTHFR Ala222Val gene polymorphism on suicidal behavior. Interestingly, a recent study reported that Ala/Val genotype of MTHFR Ala222Val gene polymorphism was significantly more frequent in healthy controls than patients with depression, suggesting that a heterozygous effect of the MTHFR Ala222Val gene polymorphism may be a protection from depression ${ }^{56}$. To our knowledge, this is the first report to show the heterozygous effect of the MTHFR Ala222Val gene on the clinical variability of schizophrenia phenotype. Alternatively, the effects of Ala222Val polymorphism on attempted suicide may also be due to linkage disequilibrium between this polymorphism and a functional polymorphism in or near the MTHFR gene. Therefore, in order to fully understand the relationship between MTHFR genetic variants and suicide attempt in schizophrenia, haplotype analysis combining multiple MTHFR genetic polymorphisms in larger samples will be needed.

Both SCZ and suicide behavior have a multifactorial and complex etiopathogenesis, arising from a combination of genetic and environmental factors ${ }^{58,59}$. We found that more symptoms (positive symptom, depressive, excitement factors), longer duration of illness, alcohol consumption, and more cigarettes smoked each day were associated with suicidal behavior in SCZ, suggesting important environmental factors in suicide behavior among SCZ patients.

There are several limitations that should be noted. First, the effect of MTHFR Val/Val genotype can be alleviated by increasing the consumption of folate and, to a degree, cobalamin ${ }^{60}$. However, we did not measure plasma folate and homocysteine levels in the subjects and did not have information on whether our subjects lacked folate food fortification or not. Second, there was a significant difference in the age between suicide attempters and non-attempters. Many studies have found that the common factors which may exacerbate folate deficiency are 
older age, male sex, and alcoholism ${ }^{61-63}$. Third, there were more female subjects in the SCZ group, which may have led to bias in the statistical analysis due to the imbalance in the number of subjects in each sex category in our current study. Fourth, it should be noted that we only found a weak association between the Ala222/Val genotype and suicide attempt in SCZ.

To summarize, in the present study, we showed no relationship between MTHFR Ala222/Val polymorphism and SCZ, but a weak association between this polymorphism and suicide attempt in SCZ. Moreover, psychological symptoms, drinking status, and smoking possibly interacted with the MTHFR gene variant and appeared to play an important role in suicide attempts among SCZ patients. However, considerable caution should be noted in the interpretation of the results. Since the sample size was small, the significant association between MTHFR Ala222/Val polymorphism and suicidal behavior may be due to multiple tests or small sample size. Further studies in a larger sample of various ethnic populations are unavoidable to investigate the interactions between these genes and the environment.

\section{Methods}

Subjects. 957 chronic inpatients with SCZ were recruited from two large psychiatric hospitals in Northern China. The inclusion criteria were as our prior studies: age 20-75 years; Han Chinese; and diagnosis of SCZ according to DSM-IV by the trained psychiatrists. All patients were of the chronic type with an illness duration of at least 5 years. Also, they had been taking stable doses of oral antipsychotic medication for at least one year prior to recruitment. Antipsychotic medication consisted mainly of prescribed drug monotherapy, including clozapine $(n=430)$, risperidone $(n=210)$, chlorpromazine $(n=69)$, sulpiride $(n=49)$, perphenazine $(n=45)$, quetiapine $(\mathrm{n}=42)$, haloperidol $(\mathrm{n}=34)$, aripirazole $(\mathrm{n}=29)$, and others $(\mathrm{n}=49)$. Average of antipsychotic dose (in chlorpromazine equivalents) was $438 \pm 407 \mathrm{mg} /$ day.

576 unrelated healthy controls were recruited through advertisement from local communities near the hospital during the same period as the patients. A research psychiatrist ruled out potential controls with Axis I disorders on DSM-IV at the present or in their lifetime after a clinical structured interview. All the healthy controls were Chinese Han population.

We carried out a physical examination and laboratory tests from all subjects. Any subjects with abnormalities in physical health were excluded. All subjects gave informed consent to participate in the study, which was approved by the Institutional Review Board of Beijing HuiLongGuan hospital. In addition, all methods were performed in accordance with Declaration of Helsinki promulgated by the National Institute of Health.

Clinical measures. PANSS was used to assess symptoms of SCZ on the same day of blood drawing. Prior to the assessment, four psychiatrists received training sessions in the use of PANSS and conducted repeated assessment tests. After training, inter-rater correlation coefficient was greater than 0.8 for the PANSS total score.

In this study, a five-factor model of SCZ symptoms was utilized, labeling symptoms as 'positive', 'negative',

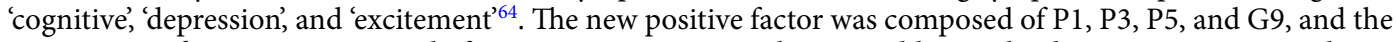
new negative factor was composed of N1, N2, N3, N4, N6, and G7. In addition, the three items P2, N5, and G11 of the PANSS scale constitute a cognitive factor, G2, G3, and G6 for a depressive factor, and P4, P7, G8, and G14 for an excitement factor.

History of suicide attempts were assessed by the same four psychiatrists based on record reviews and interview data according to a definition by $\mathrm{WHO}^{65}$. In this study, suicide attempts were defined as deliberate self-destructive behavior, with at least some intention to kill themselves, but not causing death ${ }^{65}$. During the study interview, the main question was "Have you tried to commit suicide in your lifetime?" If the answer was yes, then all subjects were asked about previous suicide attempts, including the following details: number of attempts, exact date and method of each suicide attempt. Totally, there were 152 patients who had a history of suicidal attempt (attempters) and 805 patients without history of suicidal attempt (non-attempters). The average number \pm standard deviation of attempted suicide among attempters was $1.51 \pm 0.94$ times (ranging from 1 time to 5 times).

MTHFR polymorphism analysis. Based on previous studies ${ }^{14,24}$, we selected one functional single nucleotide polymorphism Ala222 Val in the promoter of MTHFR gene. The polymorphism was genotyped following the standard procedure as described in Sequenom Genotyping Protocol and the previous studies ${ }^{66}$. The primers and extent probe of MTHFR Ala222Val were: sense: $5^{\prime}$-ACGTTGGATGCTTGAAGGAGAAGGTGTCTG -3', antisense: 5' -ACGTTGGATGCTTCACAAAGCGGAAGAATG-3' , and probe: AAGGTGTCTGCGGGAG. The sample genotyping success rate of this study averaged $98.9 \% .5 \%$ of all DNA samples were repeated, showing $99.5 \%$ reproducibility for SNP results.

Statistical analysis. Deviations from Hardy-Weinberg (HW) equilibrium were tested by using the $\chi^{2}$ test for goodness of fit. The differences in allele and genotypic frequencies of MTHFR Ala222Val polymorphism between groups were analyzed by using the $\chi^{2}$ test. Further, we used binary logistic regressions to calculate odds ratios (ORs) and their $95 \%$ confidence intervals (CIs) to evaluate the effects of different genotypes on SCZ or suicide after controlling for the confounding factors.

Analysis of variance (ANOVA) for continuous variables and the chi-squared test for categorical variables were used to analyze the differences between groups (patients vs. healthy controls or suicide attempters vs. non-attempters). The relationship between the variables is evaluated using Pearson's correlation coefficient. Bonferroni correction was used with each analysis to correct multiple tests. The variables that were significantly correlated with suicide attempt were included in the next logistic regression analysis, which was carried out to adjust for confounding factors. In the logistic regression model, the dependent variable was suicide, and the independent variables were those that were significantly different between suicide attempters and non-attempters. 
We used SPSS version 15.0 to perform all statistical analysis, with two-tailed p values of less than 0.05 . The power of the sample was calculated using Quanto software, with the relative risk and known risk allele frequencies under dominant models, respectively.

Received: 6 February 2019; Accepted: 4 November 2019; Published online: 19 March 2020

\section{References}

1. Phillips, M. R. et al. Suicide and the unique prevalence pattern of schizophrenia in mainland China: a retrospective observational study. Lancet 364, 1062-8 (2004).

2. Global, regional, and national age-sex specific all-cause and cause-specific mortality for 240 causes of death, 1990-2013: a systematic analysis for the Global Burden of Disease Study 2013. Lancet 385, 117-71 (2015).

3. Zaheer, J. et al. Service utilization and suicide among people with schizophrenia spectrum disorders. Schizophr Res 202, 347-353 (2018).

4. Chang, C. K. et al. Life expectancy at birth for people with serious mental illness and other major disorders from a secondary mental health care case register in London. PLoS One 6, e19590 (2011).

5. Tidemalm, D. et al. Familial clustering of suicide risk: a total population study of 11.4 million individuals. Psychol Med 41, 2527-34 (2011).

6. Zai, C. C. et al. Genetic Factors and Suicidal Behavior. (2012).

7. Neider, D. et al. Risk factors for suicide among patients with schizophrenia: a cohort study focused on cerebrospinal fluid levels of homovanillic acid and 5-hydroxyindoleacetic acid. Neuropsychiatr Dis Treat 12, 1711-4 (2016).

8. de Medeiros Alves, V. et al. Suicide attempt in mental disorders (MeDi): Association with 5-HTT, IL-10 and TNF-alpha polymorphisms. J Psychiatr Res 91, 36-46 (2017).

9. Jakhar, K. et al. Self-harm and suicide attempts in Schizophrenia. Asian J Psychiatr 30, 102-106 (2017).

10. Lightfoot, T. J. et al. Genetic variation in the folate metabolic pathway and risk of childhood leukemia. Blood 115, 3923-9 (2010).

11. Bahous, R. H. et al. Early Manifestations of Brain Aging in Mice Due to Low Dietary Folate and Mild MTHFR Deficiency. Mol Neurobiol (2018).

12. Tanwar, H. et al. A Computational Approach to Identify the Biophysical and Structural Aspects of Methylenetetrahydrofolate Reductase (MTHFR) Mutations (A222V, E429A, and R594Q) Leading to Schizophrenia. Adv Protein Chem Struct Biol 108, 105-125 (2017).

13. Yadav, U. et al. Role of MTHFR C677T gene polymorphism in the susceptibility of schizophrenia: An updated meta-analysis. Asian J Psychiatr 20, 41-51 (2016).

14. Zhilyaeva, T. V. et al. Association study of methylenetetrahydrofolate reductase genetic polymorphism $677 \mathrm{C}>\mathrm{T}$ with schizophrenia in hospitalized patients in population of European Russia. Asian J Psychiatr 32, 29-33 (2018).

15. El-Baz, F. et al. Study of the C677T and 1298AC polymorphic genotypes of MTHFR Gene in autism spectrum disorder. Electron Physician 9, 5287-5293 (2017).

16. Raghavan, R. et al. Maternal Multivitamin Intake, Plasma Folate and Vitamin B12 Levels and Autism Spectrum Disorder Risk in Offspring. Paediatr Perinat Epidemiol 32, 100-111 (2018).

17. Zhang, Z. et al. Association Study of Polymorphisms in Genes Relevant to Vitamin B12 and Folate Metabolism with Childhood Autism Spectrum Disorder in a Han Chinese Population. Med Sci Monit 24, 370-376 (2018).

18. Bondarenko, E. A. et al. Genetic Analysis of BDNF, GNB3, MTHFR, ACE and APOE Variants in Major and Recurrent Depressive Disorders in Russia. Int J Med Sci 13, 977-983 (2016).

19. Jimenez, K. M. et al. MTHFR gene methylation is associated with perceived stress in healthy young adults. Psychiatr Genet $\mathbf{2 8 , 4 1 - 4 6}$ (2018).

20. Rai, V. Association of C677T polymorphism (rs1801133) in MTHFR gene with depression. Cell Mol Biol (Noisy-le-grand) 63, 60-67 (2017).

21. Jha, S. et al. Effectiveness of add-on l-methylfolate therapy in a complex psychiatric illness with MTHFR C677 T genetic polymorphism. Asian J Psychiatr 22, 74-5 (2016).

22. Wan, L. et al. Methylenetetrahydrofolate reductase and psychiatric diseases. Transl Psychiatry 8, 242 (2018).

23. Xu, R. et al. Lack of association between MTHFR C677T Gene polymorphism with alcohol dependence: A meta-analysis of casecontrol studies. Neurosci Lett 683, 69-74 (2018).

24. Chojnicka, I. et al. No association between MTHFR C677T polymorphism and completed suicide. Gene 511, 118-21 (2012).

25. Yoshimi, A. et al. Gene-wide association study between the methylenetetrahydrofolate reductase gene (MTHFR) and schizophrenia in the Japanese population, with an updated meta-analysis on currently available data. Schizophr Res 124, 216-22 (2010).

26. Peerbooms, O. L. et al. Meta-analysis of MTHFR gene variants in schizophrenia, bipolar disorder and unipolar depressive disorder: evidence for a common genetic vulnerability? Brain Behav Immun 25, 1530-43 (2011).

27. Nishi, A. et al. Meta-analyses of blood homocysteine levels for gender and genetic association studies of the MTHFR C677T polymorphism in schizophrenia. Schizophr Bull 40, 1154-63 (2014).

28. Hu, C. Y. et al. Methylenetetrahydrofolate reductase (MTHFR) polymorphism susceptibility to schizophrenia and bipolar disorder: an updated meta-analysis. J Neural Transm (Vienna) 122, 307-20 (2015).

29. Zintzaras, E. C677T and A1298C methylenetetrahydrofolate reductase gene polymorphisms in schizophrenia, bipolar disorder and depression: a meta-analysis of genetic association studies. Psychiatr Genet 16, 105-15 (2006)

30. Muntjewerff, J. W. et al. Homocysteine, methylenetetrahydrofolate reductase and risk of schizophrenia: a meta-analysis. Mol Psychiatry 11, 143-9 (2006).

31. Feng, L. G. et al. Association of plasma homocysteine and methylenetetrahydrofolate reductase C677T gene variant with schizophrenia: A Chinese Han population-based case-control study. Psychiatry Res 168, 205-8 (2009).

32. Zhang, Y. et al. Association of MTHFR C677T polymorphism with schizophrenia and its effect on episodic memory and gray matter density in patients. Behav Brain Res 243, 146-52 (2013).

33. Kay, S. R. et al. The positive and negative syndrome scale (PANSS) for schizophrenia. Schizophr Bull 13, 261-76 (1987).

34. Kim, S. G. et al. No association of functional polymorphisms in methlylenetetrahydrofolate reductase and the risk and minor physical anomalies of schizophrenia in Korean population. J Korean Med Sci 26, 1356-63 (2011).

35. Kang, H. J. et al. No Association Between Functional Polymorphisms in COMT and MTHFR and Schizophrenia Risk in Korean Population. Epidemiol Health 32, e2010011 (2010).

36. Arzaghi, S. M. et al. C677T Methylenetetrahydrofolate Reductase (MTHFR) Gene Polymorphism in Schizophrenia and Bipolar Disorder: An Association Study in Iranian Population. Iran J Psychiatry 6, 1-6 (2011).

37. Jonsson, E. G. et al. Two methylenetetrahydrofolate reductase gene (MTHFR) polymorphisms, schizophrenia and bipolar disorder: an association study. Am J Med Genet B Neuropsychiatr Genet 147B, 976-82 (2008).

38. Hiraoka, M. et al. Genetic polymorphisms and folate status. Congenit Anom (Kyoto) 57, 142-149 (2017). 
39. Mandaviya, P. R. et al. Homocysteine and DNA methylation: a review of animal and human literature. Mol Genet Metab 113, 243-52 (2014).

40. Hoffman, A. et al. Methylenetetrahydrofolate Reductase Deficiency Deregulates Regional Brain Amyloid-beta Protein Precursor Expression and Phosphorylation Levels. J Alzheimers Dis 64, 223-237 (2018).

41. Christensen, B. et al. Correlation of a common mutation in the methylenetetrahydrofolate reductase gene with plasma homocysteine in patients with premature coronary artery disease. Arterioscler Thromb Vasc Biol 17, 569-73 (1997).

42. Bolton, A. D. et al. Synaptic Effects of Dopamine Breakdown and Their Relation to Schizophrenia-Linked Working Memory Deficits. Front Synaptic Neurosci 10, 16 (2018).

43. Fan, N. et al. Effect of risperidone on serum homocysteine levels in first-episode, drug-naive patients with schizophrenia. Neurosci Lett 650, 168-173 (2017).

44. Enko, D. et al. Association between increased plasma levels of homocysteine and depression observed in individuals with primary lactose malabsorption. PLoS One 13, e0202567 (2018).

45. Yin, J. et al. Elevated circulating homocysteine and high-sensitivity C-reactive protein jointly predicts post-stroke depression among Chinese patients with acute ischemic stroke. Clin Chim Acta 479, 132-137 (2018).

46. Ayesa-Arriola, R. et al. Suicidal behaviour in first-episode non-affective psychosis: Specific risk periods and stage-related factors. Eur Neuropsychopharmacol 25, 2278-88 (2015).

47. Canal-Rivero, M. et al. Predicting suicidal behaviour after first episode of non-affective psychosis: The role of neurocognitive functioning. Eur Psychiatry 53, 52-57 (2018).

48. Jing, Yl et al. The suicide attempt rate of long-term hospitalized patients with schizophrenia. Journal of Shand med 6, 105-107 (2014).

49. Sayadi, M. A. et al. CT genotype of 5,10-methylenetetrahydrofolate reductase (MTHFR) C677T polymorphism is protector factor of major depressive disorder in the Tunisian population: a case control study. Ann Gen Psychiatry 15, 18 (2016).

50. Pandya, C. et al. Glucocorticoid regulates TrkB protein levels via c-Cbl dependent ubiquitination: a decrease in $\mathrm{c}-\mathrm{Cbl} \mathrm{mRNA}$ in the prefrontal cortex of suicide subjects. Psychoneuroendocrinology 45, 108-18 (2014).

51. Rhodes, A. E. et al. Antecedents and sex/gender differences in youth suicidal behavior. World J Psychiatry 4, 120-32 (2014).

52. Zheng, L. et al. Conversion from MCI to AD in patients with the APOE epsilon4 genotype: Prediction by plasma HCY and serum BDNF. Neurosci Lett 626, 19-24 (2016).

53. Bicikova, M. et al. Physiological changes after spa treatment - a focus on endocrinology. Physiol Res 67, S525-S530 (2018).

54. Tillmann, S. et al. Probiotics Affect One-Carbon Metabolites and Catecholamines in a Genetic Rat Model of Depression. Mol Nutr Food Res 62, e1701070 (2018).

55. Bani-Fatemi, A. et al. Epigenome-wide association study of suicide attempt in schizophrenia. J Psychiatr Res 104, 192-197 (2018).

56. Wang, Q. et al. Role of Complex Epigenetic Switching in Tumor Necrosis Factor-alpha Upregulation in the Prefrontal Cortex of Suicide Subjects. Am J Psychiatry 175, 262-274 (2018).

57. Giner, L. et al. Cognitive, Emotional, Temperament, and Personality Trait Correlates of Suicidal Behavior. Curr Psychiatry Rep 18, $102(2016)$.

58. Pompili, M. et al. Genetic association analysis of serotonin and signal transduction pathways in suicide attempters from an Italian sample of psychiatric patients. Neurosci Lett 656, 94-102 (2017).

59. Lieberman, J. A. et al. Psychotic Disorders. N Engl J Med 379, 270-280 (2018).

60. Dhonukshe-Rutten, R. A. et al. Dietary intake and status of folate and vitamin B12 and their association with homocysteine and cardiovascular disease in European populations. Eur J Clin Nutr 63, 18-30 (2009).

61. Hamid, A. et al. New perspectives on folate transport in relation to alcoholism-induced folate malabsorption-association with epigenome stability and cancer development. FEBS J 276, 2175-91 (2009).

62. Waskiewicz, A. et al. Impact of MTHFR C677T gene polymorphism and vitamins intake on homocysteine concentration in the Polish adult population. Kardiol Pol 69, 1259-64 (2011).

63. Zhang, D. M. et al. Efficacy of Vitamin B Supplementation on Cognition in Elderly Patients With Cognitive-Related Diseases. J Geriatr Psychiatry Neurol 30, 50-59 (2017).

64. Rodriguez-Jimenez, R. et al. Cognition and the five-factor model of the positive and negative syndrome scale in schizophrenia. Schizophr Res 143, 77-83 (2013).

65. Krug, E. G. et al. The world report on violence and health. Lancet 360, 1083-8 (2002).

66. Jurinke, C. et al. MALDI-TOF mass spectrometry: a versatile tool for high-performance DNA analysis. Mol Biotechnol 26, 147-64 (2004).

\section{Author contributions}

Jia Hong Liu was responsible for recruiting the patients and wrote the paper. Mei Hong Xiu was responsible for study design. Xiang Yang Zhang was responsible for study design and statistical analysis, and manuscript preparation. Cheng Zhu was involved in recruiting the patients and performing the clinical rating. Wei Tang was responsible for recruiting the patients. Ke Zheng was responsible for recruiting the patients and performing the clinical rating. $\mathrm{Da}$ Chun Chen was responsible for recruiting the patients, performing the clinical rating and collecting the clinical data. Tammy H Trihn was involved in evolving the ideas. Hanjing Emily Wu was responsible for editing the manuscript. All authors have contributed to and have approved the final manuscript.

\section{Competing interests}

The authors declare no competing interests.

\section{Additional information}

Correspondence and requests for materials should be addressed to M.H.X. or X.Y.Z.

Reprints and permissions information is available at www.nature.com/reprints.

Publisher's note Springer Nature remains neutral with regard to jurisdictional claims in published maps and institutional affiliations. 
(c) (i) Open Access This article is licensed under a Creative Commons Attribution 4.0 International License, which permits use, sharing, adaptation, distribution and reproduction in any medium or format, as long as you give appropriate credit to the original author(s) and the source, provide a link to the Creative Commons license, and indicate if changes were made. The images or other third party material in this article are included in the article's Creative Commons license, unless indicated otherwise in a credit line to the material. If material is not included in the article's Creative Commons license and your intended use is not permitted by statutory regulation or exceeds the permitted use, you will need to obtain permission directly from the copyright holder. To view a copy of this license, visit http://creativecommons.org/licenses/by/4.0/.

(C) The Author(s) 2020 